\title{
Robotic stereotactic body radiation therapy for liver-limited malignant tumors
}

\author{
Mariusz Frączek ${ }^{1}$, Jacek Sobocki ${ }^{2}$, Katarzyna Pędziwiatr ${ }^{3}$, Edward Skrocki ${ }^{3}$, Norbert Piotrkowicz ${ }^{3}$, \\ Dobromira Tyc-Szczepaniak ${ }^{3}$, Elżbieta Korab-Chrzanowska ${ }^{3}$, Piotr Hevelke ${ }^{3}$, Maciej Krasnodębski ${ }^{1}$, \\ Waldemar Koszewski ${ }^{3}$ \\ ${ }^{1}$ Department of General, Transplant and Liver Surgery, Medical University of Warsaw, Warsaw, Poland \\ ${ }^{2}$ Department of General, Oncology and Gastrointestinal Surgery, St. Anna Memorial Hospital, Warsaw, Poland \\ ${ }^{3}$ Institute of CyberSurgery, Wieliszew, Poland
}

Videosurgery Miniinv 2014; 9 (4): 511-516 DOI: $10.5114 /$ wiitm.2014.44258

\begin{abstract}
Introduction: Stereotactic body radiotherapy (SBRT) is rapidly gaining favor as a new treatment modality for malignant liver tumors. Most of the studies have recruited patients with disseminated disease originating from the liver. This study focuses on disease limited to the liver.

Aim: To perform a retrospective analysis of all patients with liver tumors treated by robotic stereotactic body radiation therapy in a single center.

Material and methods: The study included 13 patients with 22 lesions. The inclusion criteria were: patients with 1-4 inoperable liver lesions and absence of any extrahepatic disease. All but 3 patients received 3 fractions delivered by the Cyberknife system of a total of 45 grey (Gy). The other 3 patients received $30 \mathrm{~Gy}$.

Results: The median follow-up time was 10.8 months (range: 7-16). The median dose was 41.5 Gy (range: 30-45). One lesion regressed (8\%). In 5 patients, the disease was locally stabilized (38\%), and in 7 other patients progression occurred (54\%). Twelve patients (92\%) are still alive, and 1 patient (8\%) died. In 1 patient a new cancer (leukemia) was diagnosed.

Conclusions: The SBRT is well tolerated and effective for local control of most liver malignant tumors. It appears that SBRT is best suited for those patients in whom systemic recurrence can be controlled by chemotherapy. Further studies are mandatory to elucidate these effects on tumors of varying histology and to elaborate upon criteria used to select patients who can benefit most from this treatment.
\end{abstract}

Key words: cyberknife, liver tumors, stereotactic body radiotherapy.

\section{Introduction}

Liver surgery is a broadly accepted radical treatment of primary liver tumors and several metastatic cancers. These include colorectal cancer and neuroendocrine tumors [1]; however, the size and location of the tumor, as well as patient condition, limit the use of this method in a large group of patients [2].
The former group has been treated with some success by radiofrequency ablation (RFA) [3]. The RFA is also limited by size, location of a tumor (for example, proximity to the liver surface or to large vessels) and breathing motion. Implementation of the automated registration of breathing motion can be applied to compensate only for the former problem [4]. Several patients were beyond the reach of local treatment.

\section{Address for correspondence}

Mariusz Frączek MD, PhD, Department of General, Transplant and Liver Surgery, Medical University of Warsaw, 16/20 Barska St,

02-315 Warsaw, Poland, phone: +48 601329 250, e-mail: mariusz.fracz@gmail.com 
Robotic stereotactic body radiation therapy (SBRT) was proposed for the treatment of liver metastases of different origin [5] and primary tumors [6]. The data from the literature suggest that local control is achieved in $90 \%$ and $86 \%$ of cases, effective for 1 and 2 years, respectively [7]. A complete response was achieved in $66 \%$ of patients, and a partial response in $13 \%$ of lesions treated. It has been reported that 1- and 2-year survival reaches 94\% and $48 \%$, respectively.

\section{Aim}

The aim of this study was to perform a retrospective analysis of all patients with liver tumors treated by robotic stereotactic body radiation therapy in a single center.

\section{Material and methods}

The retrospective analysis covered all patients treated for malignant liver tumors between July 2012 and January 2013. The indication for SBRT was determined by a multidisciplinary team which consisted of a hepatic surgeon, oncologist, radiotherapist and radiologist. Inclusion criteria comprised the following: 1 to 4 lesions located close enough to one another to enable simultaneous irradiation, diameter of the greatest lesion less than $100 \mathrm{~mm}$, and no evidence of dissemination of the disease outside of the liver. Thirteen patients with 22 lesions were treated. The mean age of the patient was 64 years old (range: 48-86), and the mean time from surgery to the SBRT was 16.6 months. Demographics of the patients and origin of the tumor are shown in Table I. Exclusion criteria are presented in Table II. Presence or suspicion of extrahepatic disease was considered an exclusion criterion. For this purpose all patients underwent a positron emission tomography-computed tomography (CT-PET) study. Mean follow-up was 10.8 months (range: 7-16 months).

Gross tumor volume (GTV) was estimated using contrast-enhanced tomography scans. Gold seed markers (fiducials) were placed around the tumor. Care was taken not to put markers in the same transversal plane to each other, nor to place them inside the tumor, so as to prevent interference with the measurements of the lesion in the control CT study.

Treatment was planned at least 7 days after implantation of the fiducials. The irradiation field was determined using CT scans in the treatment position, while the patient was immobilized using a customized mattress. According to the protocol used in

Table I. Demographic data and origin of the tumor

\begin{tabular}{|c|c|c|c|c|c|}
\hline No. & Age [years] & Gender & Origin of cancer & Previous surgery & Time from surgery to SBRT [months] \\
\hline 1 & 66 & Male & CRC & Left hemicolectomy & 10 \\
\hline 2 & 85 & Male & CRC & Left hemicolectomy & 14 \\
\hline 3 & 77 & Male & $\mathrm{HCC}$ & None & $6^{a}$ \\
\hline 4 & 59 & Male & CRC & Anterior rectal resection & 3 \\
\hline 5 & 79 & Female & CRC & Anterior rectal resection & 60 \\
\hline 6 & 70 & Female & $\mathrm{HCC}$ & None & $4^{a}$ \\
\hline 7 & 57 & Female & GC & Gastrectomy & 24 \\
\hline 8 & 70 & Male & CRC & Left hemicolectomy & 10 \\
\hline 9 & 54 & Female & $A C$ & Adrenalectomy & 18 \\
\hline 10 & 47 & Male & CRC & Left hemicolectomy & 14 \\
\hline 11 & 46 & Female & $\mathrm{GC}$ & Gastrectomy & 2 \\
\hline 12 & 71 & Male & CRC & Anterior rectal resection & 14 \\
\hline 13 & 48 & Male & LS & Tumorectomy & 13 \\
\hline
\end{tabular}

$C R C$ - colorectal cancer, HCC - hepatic cell cancer, GC - gastric cancer, LS - retroperitoneal liposarcoma AC - adrenal gland cancer, ${ }^{a}$ time from diagnosis 
other centers, GTV was calculated for contrast-enhancing disease visible on the CT scan in 1.25- $\mathrm{mm}$ slice thickness (Photo 1). The clinical target volume (CTV) was contoured by adding a $5 \mathrm{~mm}$ margin to cover possible microscopic spread. Finally, the planning target volume (PTV) was determined adding an additional $3 \mathrm{~mm}$ margin for targeting uncertainty of movement.

The treatment was performed using the CyberKnife (Accuray, USA). Ten patients received 3 fractions of $15 \mathrm{~Gy}, 2$ received 3 fractions of $10 \mathrm{~Gy}$, and 1 received 6 fractions of $5 \mathrm{~Gy}$. A control contrast-enhanced tomography study was performed after 3 and 6 months.

Regression of tumors was assessed according to mRECIST criteria.

\section{Results}

The average total dose per patient was $40.8 \mathrm{~Gy}$, with maximum $45 \mathrm{~Gy}$, minimum $30 \mathrm{~Gy}$ and median
Table II. Exclusion criteria

\begin{tabular}{|lc|}
\hline No & Criteria \\
\hline 1 & Size of lesion $>100 \mathrm{~mm}$ \\
\hline 2 & Number of lesions $>4$ \\
\hline 3 & Extra-hepatic disease \\
\hline 4 & ECOG $>3$ \\
\hline 5 & $18<$ Age $>85$ \\
\hline 6 & Direct contact of lesion with the bowel \\
\hline
\end{tabular}

45 Gy. Total dose, number of fractions, size of tumor, and gross tumor volume, before and after treatment, in consecutive patients, are presented in Table III. In 1 patient migration of the gold fiducial was observed outside of the hepatic capsule, requiring subsequent re-implantation.

We observed limited toxicity associated with the treatment. One patient (no. 3 in Tables I and III) with hepatocellular carcinoma complained of nausea and

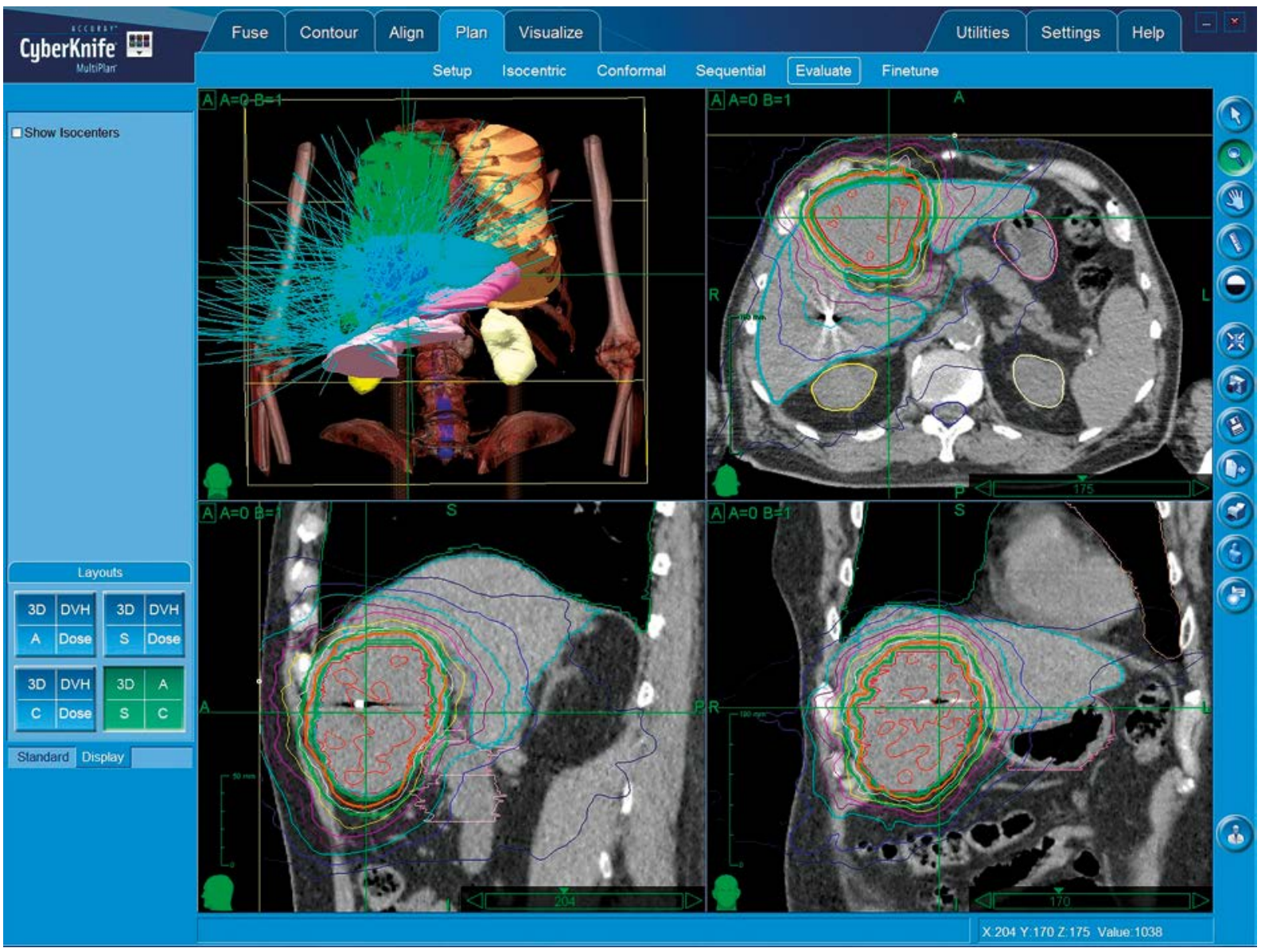

Photo 1. Planning of treatment 


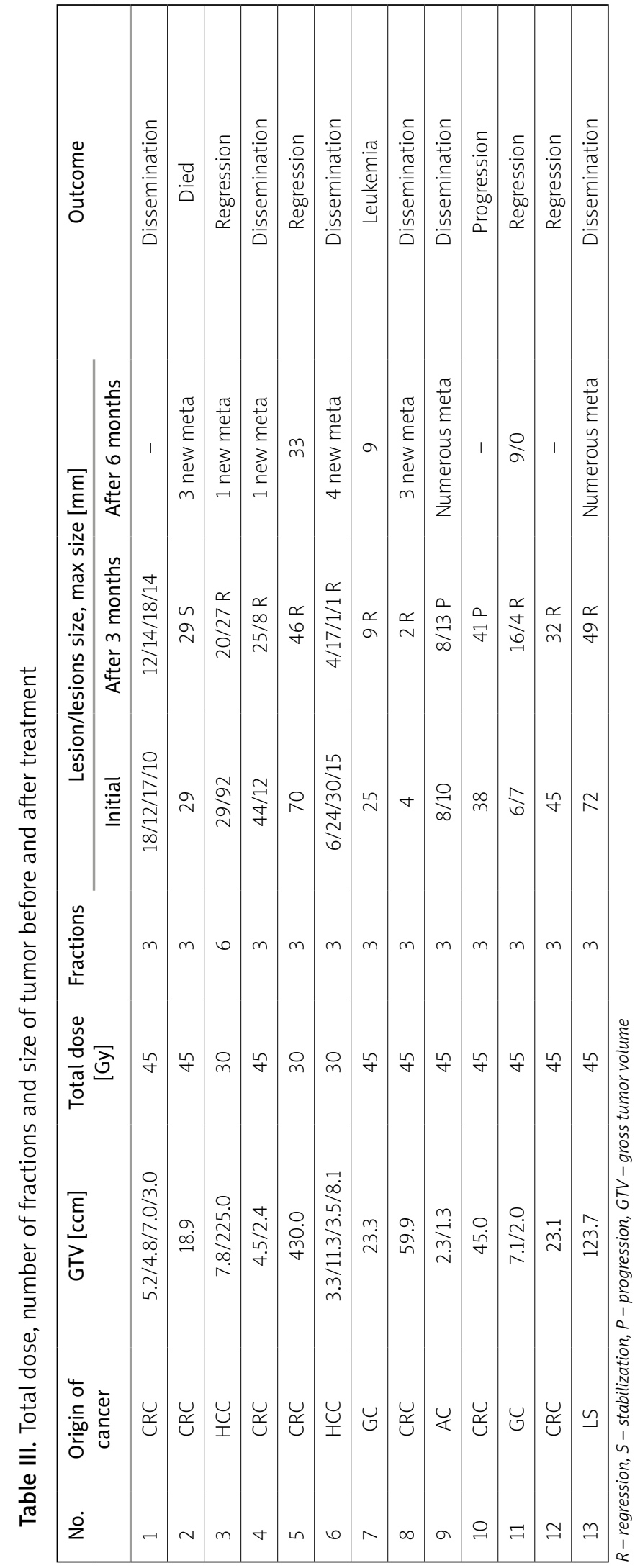

vomiting after the second course of radiation. There were no other complaints regarding toxicity-associated symptoms.

We observed an objective tumor response in 16 of 22 metastases (72\%: 1 complete, 15 partial responses) and stable disease in 1 patient (5\%). Five lesions (23\%) progressed within 3 months of receiving treatment. The regression in 1 patient was visualized in magnetic resonance imaging before and after treatment (Photos 2 and 3 respectively).

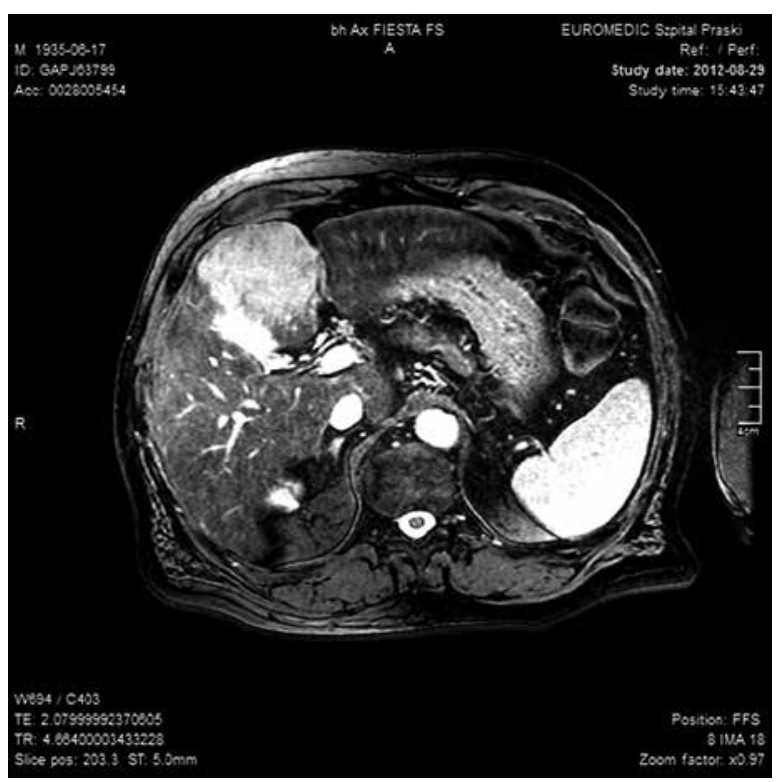

Photo 2. Magnetic resonance images showing tumor before treatment

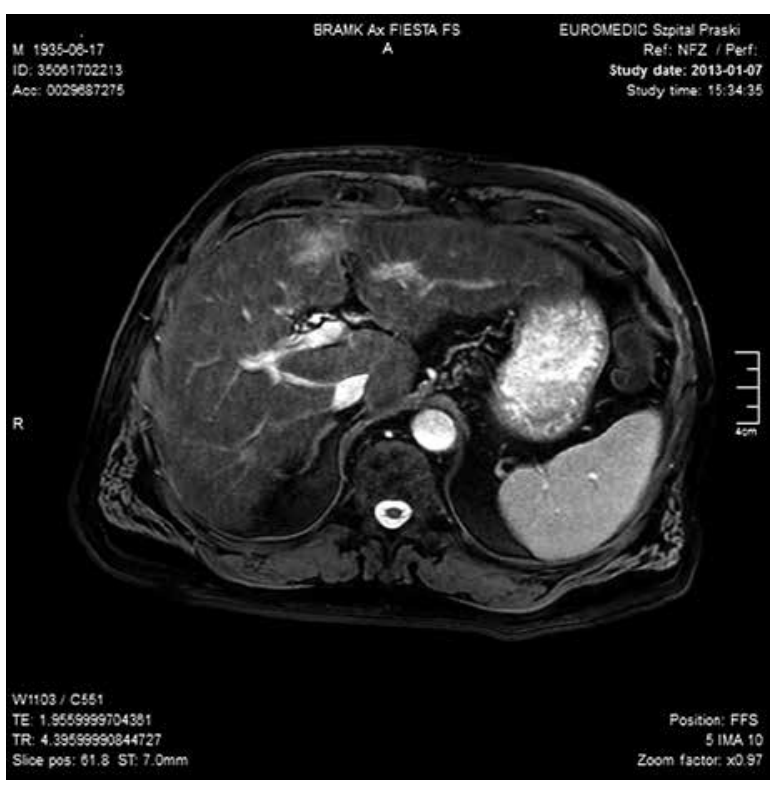

Photo 3. Regression of tumor 
Seven patients developed new metastases in the liver within 3 months. Similarly to other papers, we observed the best response, complete regression, in 1 of 2 gastric cancer patients. One patient died, 1 developed a new cancer (leukemia), and 7 developed dissemination of the disease to other organs within 6 months. Only 4 patients demonstrated a clear benefit from the treatment in the observation period, defined as local control of disease without any signs of loco-regional recurrence or distant metastases. In 2 of 7 patients with colorectal cancer metastases, we observed moderate regression of lesions. This was defined as a reduction in their dimensions. One case of liposarcoma was treated. The objective local response was partial, but numerous metastases in other locations reduced the enthusiasm for continuing with this patient's radiotherapy plan. Table IV presents the type of chemotherapy concomitant to SBRT introduced in respective patients.

\section{Discussion}

The SBRT is a relatively novel, minimally invasive technique adapted for the treatment of liver malignant lesions. It's advantage is local ablation of tumors using precise and hypofractionated irradiation. We observed regression in 9 of 13 patients, in 1 patient the tumor size was stabilized, and in 3 patients tumor progression was found.

\section{Histology-related response}

We have treated 7 patients with colorectal metastases and 6 patients with other histological type tumors. Lee et al. suggest that colorectal liver metastases appear to have weakened local control in comparison with others, but these results are not significant due to the small number of observed cases [7]. In our study metastases regressed in 4 of 7 patients with colorectal cancer. The best response was observed in patients suffering from gastric cancer. All metastases in this group regressed while one disappeared. This is in concordance with results published by other authors [8]. Several papers have been published on the treatment of hepatocellular carcinoma by SBRT [9-11]. We have treated 2 patients with this type of tumor. Similarly to most papers, in both cases we observed regression of the primary tumor. Unfortunately, metastases out of the irradiated field developed in both patients.
Table IV. Chemotherapy concomitant to SBRT

\begin{tabular}{|c|c|}
\hline No. & Concomitant chemotherapy \\
\hline 1 & FOLFIRI (leucovorin, 5-fluorouracil, irinotecan) 12 cycles \\
\hline 2 & Oxaliplatin + capecytabine 8 cycles \\
\hline 3 & No concomitant chemotherapy \\
\hline 4 & $\begin{array}{l}\text { FOLFOX (oxaliplatin, 5-fluorouracil, leucovorin) } \\
\text { + Erbitox (cetuximab) } 4 \text { cycles }\end{array}$ \\
\hline 5 & $5 F u+$ LV (5-fluorouracil z leucovorin) 6 cycles \\
\hline 6 & Interferon \\
\hline 7 & No concomitant chemotherapy \\
\hline 8 & Disqualified from chemotherapy \\
\hline 9 & Mitotane \\
\hline 10 & No concomitant chemotherapy \\
\hline 11 & No concomitant chemotherapy \\
\hline 12 & Disqualified from chemotherapy \\
\hline 13 & No concomitant chemotherapy \\
\hline
\end{tabular}

\section{Stereotactic body radiation therapy-induced tumor regression}

Fifteen of 22 tumors regressed in our group of patients, a $68 \%$ response. This is a much weaker response than reported local control rates published by other authors. One-year response rates from different studies range from $71 \%$ to $95 \%$ [12-20]. The impact of tumor size on outcome remains unclear in the literature. Vautravers-Dewas et al. did not observe any statistical difference in degree of regression between tumors larger or smaller than $25 \mathrm{~mm}$ [12]. Similar results have been published by Herfarth et al. [13]. In contrast to these data, Rusthoven et al. suggest that lesions with a maximum diameter of $<3 \mathrm{~cm}$ had a 2-year local control rate of $100 \%$ compared with $77 \%$ for lesion diameters of $>3 \mathrm{~cm}(p=0.015)$ [14]. The SBRT-induced regression was observed in most studies [15-20]; however, the principal problem in the comparative analysis of the published data is the variation in response criteria.

\section{Toxicity of therapy}

We observed only a mild adverse event in $1 \mathrm{pa}-$ tient. This was nausea and vomiting at the beginning of irradiation which resolved spontaneously and never returned. This means that only $7.6 \%$ of the group studied complained of symptoms associated with 
mild toxicity. There were no adverse events reported in this investigation. Vautravers-Dewas et al. observed a greater incidence of adverse events of the therapy, including nausea, vomiting, gastritis, gastric ulceration, esophagitis, anorexia, diarrhea/constipation, hepatic pain, cirrhotic decompensation, asthenia and pericardial effusion. Of these, nausea was most frequent and occurred in $27.6 \%$ and $25 \%$ of cases for 40 and $45 \mathrm{~Gy}$, respectively [12].

\section{Survival}

All but 1 patient survived 1 year, accounting for $93 \%$ of our group. Similar results are published by Vautravers-Dewas et al. [12]. The same author published a 2-year survival rate of $48 \%$. In contrast to our study, patients were included with metastatic disease not confined to the liver $35.7 \%$ at the time of SBRT). One-year survival counted for specific cancers was as follows: colorectal cancer 6 of 7, hepatocellular carcinoma 2 of 2, gastric cancer 2 of 2 , liposarcoma 1 of 1 , and adrenal cancer 1 death of 1 treated patient.

\section{Conclusions}

The SBRT is a well-tolerated method and is effective for local control of some subgroups of malignant liver tumors. It appears that SBRT is most appropriate for use in those patients in whom systemic recurrence can be controlled by adjuvant chemotherapy. Further studies are mandatory to elucidate the effect on tumors of various histology and to elaborate criteria to select patients who can profit from the treatment.

\section{References}

1. Gallinger S, Biagi JJ, Fletcher GG, et al. Liver resection for colorectal cancer metastases. Curr Oncol 2013; 20: e255-65.

2. Shah K, Clary B. Synchronous colorectal liver metastases: the "surgeon as oncologist" perspective. Minerva Chir 2013; 68 49-76.

3. Karanicolas PJ, Jarnagin WR, Gonen M, et al. Long-term outcomes following tumor ablation for treatment of bilateral colorectal liver metastases. JAMA Surg 2013; 148: 597-601.

4. Spinczyk D, Zylkowski J, Wroblewski T. Continuous registration on computed tomography for breathing motion compensation. Videosurgery Miniinv 2013; 8: 265-72.

5. Carey Sampson M, Katz A, Constine LS. Stereotactic body radiation therapy for extracranial oligometastases: does the sword have a double edge? Semin Radiat Oncol 2006; 16: 67-76.

6. Choi BO, Choi IB, Jang HS, et al. Stereotactic body radiation therapy with or without transarterial chemoembolization for patients with primary hepatocellular carcinoma: preliminary analysis. BMC Cancer 2008; 8: 351-9.

7. Lee MT, Kim JJ, Dinniwell R, et al. Phase I study of individualized stereotactic body radiotherapy of liver metastases. J Clin Oncol 2009; 27: 1585-91.

8. Katayanagi S, Shimazu M, Konno O, et al. A case of liver metastases from gastric cancer treated with stereotactic body radiation therapy. Gan To Kagaku Ryoho 2011; 38: 2097-9.

9. Hoffe SE, Finkelstein SE, Russell MS, Shridhar R. Nonsurgical options for hepatocellular carcinoma: evolving role of external beam radiotherapy Cancer Control 2010; 17: 100-10.

10. Kwon JH, Bae SH, Kim JY, et al. Long-term effect of stereotactic body radiation therapy for primary hepatocellular carcinoma ineligible for local ablation therapy or surgical resection. Stereotactic radiotherapy for livercancer. BMC Cancer 2010; 10: 475-84.

11. Son SH, Choi BO, Ryu MR, et al. Stereotactic body radiotherapy for patients with unresectable primary hepatocellular carcinoma: dose-volumetric parameters predicting the hepatic complication. Int J Radiation Oncology Biol Phys 2010; 78: 1073-80.

12. Vautravers-Dewas C, Dewas S, Bonodeau F, et al. Image-guided robotic stereotactic body radiation therapy for liver metastases: is there a dose response relationship? Int I Radiation Oncology Biol Phys 2011; 81: 39-47.

13. Herfarth KK, Debus J, Lohr F, et al. Stereotactic single-dose radiation therapy of liver tumors: results of a phase I/II trial. J Clin Oncol 2001; 19: 164-70.

14. Rusthoven KE, Kavanagh BD, Cardenes H, et al. Multi-institutional phase I/II trial of stereotactic body radiation therapy for liver metastases. J Clin Oncol 2009; 27: 1572-8.

15. Sato M, Uematsu M, Yamamoto F, et al. Feasibility of frameless stereotactic high-dose radiation therapy for primary or metastatic liver cancer. J Radiosurg 1998; 1: 233-8.

16. Wulf J, Hadinger U, Oppitz U, et al. Stereotactic radiotherapy of targets in the lung and liver. Strahlenther Onkol 2001; 177 : 645-55.

17. Wada H, Takai Y, Nemoto K, Yamada S. Univariate analysis of factors correlated with tumor control probability of three- dimensional conformal hypofractionated high-dose radiotherapy for small pulmonary or hepatic tumors. Int J Radiat Oncol Biol Phys 2004; 58: 1114-20.

18. Schefter TE, Kavanagh BD, Timmerman RD, et al. A phase I trial of stereotactic body radiation therapy (SBRT) for liver metastases. Int J Radiat Oncol Biol Phys 2005; 62: 1371-8.

19. Katz AW, Carey-Sampson M, Muhs AG, et al. Hypofractionated stereotactic body radiation therapy (SBRT) for limited hepatic metastases. Int J Radiat Oncol Biol Phys 2007; 67: 793-8.

20. Milano MT, Katz AW, Muhs AG, et al. A prospective pilot study of curative-intent stereotactic body radiation therapy in $\mathrm{pa}$ tients with 5 or fewer oligometastatic lesions. Cancer 2008; 112: 650-8.

Received: 10.03.2014, accepted: 17.06.2014 